\title{
Statistical Approach Involving Bayes' Theorem and the Estimation of the Prior Distribution
}

\author{
Hirosi Hưdimoto
}

The Institute of Statistical Mathematics, Tolyo

\section{Historical background}

The origins of various methods of statistical inference which are now widely used can be traced back for about 200 years. However, differences of opinion about the validity of the methods proposed occasionally have arisen from the variety of possible viewpoints. Most statisticians like to set up a statistical model, the parent population, of the statistical phenomenon using concepts of probability theory. However, statistical inference is not to develop such a deductive logic as mathematics but to intend to perform an inductive inference. Then, the statistical model should be changed to the inductive form based on given statistical evidence in the performance of statistical inference. The Bayes'theorem published in 1763 originally aimed to explain this situation. It can be expressed in the form

$$
\omega(\theta \mid x) \propto \omega(\theta) f(x \mid \theta),
$$

where $\omega(\theta)$ is the initial or prior probability distribution of the parameter $\theta, f(x \mid \theta)$ is the probability distribution of the measurement $x$ when $\theta$ is given, and $\omega(\theta \mid x)$ is the final or posterior probability distribution of $\theta$ for the observed $x$.

Any logical model for statistical inference is usually given by specifying a parameter space $Q=\{\theta\}$, a sample space $R=\{x\}$, and a family of probability distributions $\{f(x \mid \theta)\}$. The parameter space is a description of the range of unknown parameter values $\theta$ or of statistical hypotheses under consideration, and the sample space denotes the range of possible outcomes $x$. We assume that each of the possible distributions of $X$ is labeled by a parameter point $\theta$ in a specified parameter space $\Omega$ and is represented by a specified elementary probability function $f(x \mid \theta)$. The probability of the event that yields an outcome $x$ in $E$ is

$$
P(X \in E \mid \theta)=\int_{E} f(x \mid \theta) d \mu,
$$

where $\mu$ is a specified $\sigma$-finite measure on $R$, and $E$ is any measurable set. As an additional knowledge to this model, if $\omega(\theta)$ is completely known, $f(x \mid \theta)$ can be changed to $\omega(\theta \mid x)$ by means of twisting between $x$ and $\theta$ by application of Bayes' theorem in order to obtain the probability evidence with respect to the unknown parameter value based on the observed $x$. 
For descriptive simplicity, we assume that $f(x \mid \theta)$ belongs such a family of probability distributions as a minimal sufficient statistic for $\theta$ exists and that $\theta$ is a single unknown parameter and has an absolutely continuous prior distribution with density $\omega(\theta)$ on $\Omega$. Let $X^{(n)}=\left(X_{1}, \ldots, X_{n}\right)$ be a random sample of size $n$ from $f(x \mid \theta)$ and let $t\left(X^{(n)}\right)$ be the minimal sufficient statistic for $\theta$ which has the sample distribution with density $g(t \mid \theta)$. Then, $X^{(n)}$ has the joint density $f_{n}\left(x^{(n)} \mid \theta\right)=I_{k-1}^{n} \times$ $f\left(x_{k} \mid \theta\right)$ with respect to the product measure $\mu^{(n)}=\mu \times \ldots \times \mu$ on $R_{n}$, and $\theta$ has an absolutely continuous posterior distribution with desity

$$
\omega_{n}\left(\theta \mid x^{(n)}\right)=\omega(\theta) f_{n}\left(x^{(n)} \mid \theta\right) / f_{n}\left(x^{(n)}\right),
$$

where

$$
f_{n}\left(x^{(n)}\right)=\int_{a} \omega(\theta) f_{n}\left(x^{(n)} ; \theta\right) d \theta .
$$

However, since $t\left(X^{(n)}\right)$ is the minimal sufficient statistic,

$$
\omega_{n}\left(\theta \mid x^{(n)}\right)=\pi(\theta \mid t),
$$

where

$$
\pi(\theta \mid t)=\omega(\theta) g(t \mid \theta) / \int_{\Omega} \omega(\theta) g(t \mid \theta) d \theta
$$

in $t=t\left(x^{(n)}\right)$. If we select such an interval $\left(\hat{\theta}_{1}, \hat{\theta}_{2}\right)$ as satisfies

$$
P\left(\hat{\theta}_{1}<\theta<\hat{\theta}_{2} \mid t\right)=\int_{\hat{\theta}_{1}}^{\theta_{2}} \pi(\theta \mid t) d \theta=\eta
$$

and minimizes $\left(\hat{\theta}_{2}-\hat{\theta}_{1}\right),\left(\hat{\theta}_{1}, \hat{\theta}_{2}\right)$ is a (Bayesian) confidence interval for $\theta$ with confidence probability $\boldsymbol{\eta}$.

However, these applications of Bayes' theorem have often been severely criticized because $\omega(\theta)$ was not alwayes available, and a growing tendency to avoid this kind of argument has appeared. Especially, in 1930, R.A. Fisher introduced the concept of a fiducial probability distribution for a parameter in order to make an attempt in this direction for the case of unknown $\omega(\theta)$. Fisher's fiducial method has been left over nuisance problems to interpret by the concepts of usual probability, and Neyman's confidence method was presently proposed. The first version of J. Neyman for the confidence method was that the two methods were originally supposed to be the same, but Fisher denounced it as a distortion of his original idea. The fiducial method and the confidence method usually give the same result in the case of a single parameter but occasionally different results in the case that there is more than one. The controversy related to derivation of the fiducial distribution for the difference between means of two normal populations with unknown different variances, the Behrens-Fisher problem, eventually 
has come to differentiate the two methods.

The fiducial method has been frequently criticized adversely in the literature, particularly in recent years. Fisher has devoted considerable space to his fiducial method and stated his view-points in his book, [4]. He states that an essential ingredient for its use is the absence of prior information concerning the value of the parameter being estimated.

It must be noticed that the Bayesian confidence interval for $\theta$ derived in (1.7) is a fixed interval for an observed $x^{(n)}$. On the other hand, the Neyman's confidence method can exactly make the probability statement which has a frequency interpretation in terms of a well-defined kind of repeated sampling only when it is a random interval. However, this kind of statement becomes to be deprived the exact meaning for the probability statement as soon as the interval is actually calculated for the observed $x^{(n)}$ and fixed for a practical occasion. I suppose that the word "confidence coefficient" explains an actual feeling in such situations.

Fisher descrived "the fiducial argument uses the observations (only) to change the logical status of the parameter from one in which nothing is known of it, and no probability statement can be made, to the status of a random variable having a well defined distribution", and he emphasized the necessity of basing the fiducial distribution on a sufficient statistic and more immediately on a pivotal quantity. Then, the fiducial method intended to make the same kind of probability statement for the observed value of the sufficient statistic put into the pivotal quantity. It seems to be rather similar to the Bayesian approach than the confidence method. But, the non-uniqueness of fiducial distributions in certain problems and the lack of proper relationship with prior distributions were criticized. Lately, D.A.S. Fraser, [5], tried to justify these problems for the case with the restriction of invariance.

\section{Bayesians}

The thought of the confidence method seems to be suitable in order to handle phenomena that can be observed by repeated samplings, but if a well defined distribution for the parameter could be obtained for the observed value in the general way, it would be more convenient. However, the problem as described above has arisen because of unknown prior distributions. Thus, it will be worthwhile to ask how to bring prior distributions in statistical inference. I suppose that the approach is classified into three kinds: (i) empirical Bayes approach, (ii) Bayesian approach and (iii) neo-Bayesian approach.

The approach (iii) is taken by the adherents of subjective probability which stems from F.P. Ramsey, [20] and leading representatives of the subjective view 
are L.J. Savage, [25] and I.J. Good, [6]. The approach (ii) seems to be mainly supported by British Bayesians. Some statistician of them clearly refuses to call as "Baysian" any statistician intending to apply Bayes theorem in statistical inference. R.L. Plackett [17] has stated "In view of such applications it seems a misnormer to use the term "Bayesian" for an approach involving Bayes's theorem, because this is a tool which has occasional value for any statistician. However, the term "Bayesian" can profitably be retained for individuals who take the view that probability is a numerical measure of degree of belief, since they will inevitably need Bayes's theorem in order to convert prior degree of belief, taken with the evidence supplied by the data, into posterior degress of belief." His criticism mainly has thrown toward frequentists. Then, it can be considered that not only the method of approach but also the thought is different between (i) and (ii).

The concept of a logical probability was introduced by J.M. Keynes, [13]. H. Jeffreys supported Keynes' viewpoint and he has dealt with its mathematical formulation, [12]. Jeffreys' thought largely has contributed to British Bayesian. Then, the last part of Plackett's description has asserted that they must be expressed by the concept unified by the logical probability because a prior distribution and a corresponding posterior distribution denote only different aspects of same hypothesis on a phenomenon. Thus, an assessment of the prior distribution must carry out in the premeditated logical manner according to the Bayesian viewpoint. Jeffreys' invariance theory postulates that the prior distribution is chosen by a rule which is consistent in the sense that equivalent results are obtained under any non-singular transformation of the parameters. The main proposal is for a prior density

$$
\omega(\theta) \propto|I(\theta)|^{1 / 2},
$$

where $I(\theta)$ is Fisher's imformation matrix, with elements

$$
I_{\nu \mu}(\theta)=-\varepsilon\left\{\partial^{2} \log f(x \mid \theta) / \partial \theta_{\nu} \partial \theta_{\mu}\right\} .
$$

For the viewpoint of converting the prior distribution to the posterior distribution by applying Bayes' theorem, I suppose to involve a certain intuitive motivation in setting up the prior distribution of the parameter: That is, the premeditated prior distribution must produce the similar type of probability distribution for the corresponding posterior distribution. The other technique to this inclination is the construction of conjugate families that was suggested by G.A. Barnard, [1]. Its detailed account was given by $H$. Raiffa and R. Schlaifer, [19].

D.V. Lindley discussed the conditions for a fiducial distribution of $\theta$ given $x$ to be a Bayes' distribution (posterior distribution) and obtained the result concerning location parameter probelm in the case that a one-dimensional random 
variable $X$ has a probability distribution depending on a single parameter $\theta$, where $x$ is a observed value of $X,[14]$. The problem was also discussed by Fraser from his view afterwards, [5]. The relationship between confidence distributions (or fiducial distributions) and Bayes' distributions has been further examined by B.L. Welch and H.W. Peers, [27], Welch, [28] and others and they have shown that in the case of large sample, two approaches led to very similar results under certain conditions. Now, suppose that $X^{(n)}$ is a random sample from one stated above and has the joint probability distribution with density $f_{n}\left(x^{(n)} \mid \theta\right)$. Consider that

$$
\frac{\int_{-\infty}^{h\left(x_{1}^{(n)} \eta\right)} f_{n}\left(x^{(n)} \mid \theta\right) w(\theta) d \theta}{f_{n}\left(x^{(n)}\right)}=,
$$

where $\left.f_{n}\left(x^{(n)}\right)=\int_{-\infty}^{\infty} f_{n}\left(x^{(n)}\right\} \theta\right) w(\theta) d \theta$ and $w(\theta)$ is a positive weight function. For a Bayes' distribution with the prior distribution $w(\theta)$, write (1.10) as

$$
P\left(\theta<h\left(x^{(n)}, \eta\right) \mid x^{(n)}\right)=\eta .
$$

On the other hand, in the case that $\theta$ is a unknown parameter but not random, when $h\left(x^{(n)}, \eta\right)$ and $\eta$ of (1.11) are used in the formula of confidence method, Welch and Peers, [27] show that

$$
P\left(\theta<h\left(X^{(n)}, \eta\right) \mid \theta\right)=\eta+O\left(n^{-1 / 2}\right),
$$

and particularly, for $w(\theta)=I^{1 / 2}(\theta)$

$$
P\left(\theta<h\left(X^{(n)}, \eta\right) \mid \theta\right)=\eta+O\left(n^{-1}\right) .
$$

Bayesian approaches are convenient for reconciliatory arguments. An instance of them is the problem of robustness which investigates the effect on inference of changing the distribution from $f(x \mid \theta)$ to $f(x \mid \theta, \phi)$, where $\phi$ is a nuisance parameter. However, in Bayesian approach, the prior distribution of $\phi$ is postulated from rather arbitrary viewpoints and not usually substantiated by the nature of the problem considered. J. Neyman criticized this point and he described "it is dogmatic and solves no problem", in [16]. It seems to me that in Bayesian thought, there is a inclination to conservative use of prior knowledge centered in ignorance in order to avoid tragic results.

Thus, the prior distribution should be emprically inferred if possible. The Bayesian may possibly disapprove of such a view. However, the following theorem in Lindley's recent book, [15] is such an instance as is desirable to be tested on the basis of experimental evidence: When a size $\mathrm{n}$ of random sample taken from a population distribution tends to infinity, the posterior distribution of a parameter $\theta$ is asymptotically normal with mean equal to the maximum- 
likelihood estimate and variance equal to the reciprocal of the second derivative of the logarithm of the likelihood function evaulated at the maximum- likelihood estimate. But this theorem seems to be somewhat unreal to me. The reason is as follows: Why can a random sample be infinitely taken from a fixed probability distribution without limitation of size, notwithstanding that any prior distribution has a positive density in some range of parameter values? It must be tested on the basis of experimental evidence in order to make up such a situation as this theorem is applciable.

\section{The empirical Bayes' problem}

The other approach (i) was originally proposed by H. Robbins. J. Neyman applaudingly mentioned it as "the breakthrough on the Bayes' front relating to the utilization of previous experience", [16].

Consider a random variable $X$ which has a probability distribution with $f(x \mid \theta)$ depending on an unknown real parameter $\theta$. Let $\theta$ itself be a random variable with a prior distribution function $W(\xi)=P(\theta \leqq \xi)$. Then, such the estimate $\hat{\theta}_{W}(X)$ of $\theta$ as minimizes $\varepsilon(\hat{\theta}(X)-\theta)^{2}$ is the Bayes estimate corresponding to $W(\xi)$ given by

$$
\hat{\theta}_{w}(X)=\frac{\int_{\Omega} \xi f(x \mid \xi) d W(\xi)}{\int_{\Omega} f(x \mid \xi) d W(\xi)}
$$

where $\varepsilon$ denotes the expectation with respect to the joint probability distribution of $X$ and $\theta$. However, if $W(\theta)$ is unknown, $\hat{\theta}_{W}(X)$ is not calculable.

Suppose $\left\{\left(X_{k}, \theta_{k}\right)\right\}$ to be an independent sequence of $(X, \theta)$, where $X$ is observable and $\theta$ unobservable. When $X$ is a random variable assumed to have discrete values $x$, which has a probability distribution of known form, and $W(\theta)$ is fixed but unknown, the problem of estimating $\theta_{n}$ based on the set of observed values of $X_{1}, \ldots, X_{n}$ was considered by $H$. Robbins as follows, [23]. For instance, in the case of the Poisson distribution

$$
f(x \mid \xi)=e^{-\xi} \frac{\xi x}{x !}, \quad x=0,1, \cdots, \xi>0,
$$

we have

$$
f(x)=\int f(x \mid \xi) d W(\xi)=\int_{0}^{\infty} e^{-\xi \xi x} d W(\xi) / x !
$$

and

$$
\hat{\theta}_{w}(x)=(x+1) f(x+1) / f(x) .
$$


Define the function

(1.19) $\hat{\theta}_{n}(x)=(x+1) \frac{\text { number of terms } X_{1}, \cdots, X_{n} \text { which are equal to } x+1}{\text { number of terms } X_{1}, \cdots, \bar{X}_{n} \text { which are epual to } x}$.

Then, no matter what the unknown $W(\xi)$, we have for any $x$,

$$
\hat{\theta}_{n}(x) \rightarrow \hat{\theta}_{w}(x) \text { with probability } 1 \text { as } n \rightarrow \infty \text {. }
$$

H.Robbins has further delt with some more general problems on the empirical Bayes approach in [24]. I have dealt with an embodiment of Robbins'idea in [9].

\section{The estimation of the prior distribution}

Let a random variable $X$ be known to have one of a finite number of specified distribution functions $F_{1}(x), \ldots, F_{r}(x)$ depending on the respective values of a random parameter $\theta$ in the following manner, i.e. $P(X \leqq x \mid \theta=i)=F_{i}(x)$ and $P(\theta=i)$ $=\omega_{i}$ for $i=1, \ldots, r$. Consider an independent sequence $\left\{\left(X_{k}, \theta_{k}\right)\right\}$ of pairs of random variables $(X, \theta)$. However, each $X_{k}$ is observable but every $\theta_{k}$ is unobservable. We shall deal with estimating the unknown prior distribution $\omega^{\prime}=\left(\omega_{1}, \ldots\right.$, $\left.\omega_{r}\right)$ when $X^{(n)}=\left(X_{1}, \ldots, X_{n}\right)$ is obtained. In this case, $X^{(n)}$ is considered as a random sample from

$$
F(x)=\sum_{i=1}^{r} \omega_{i} F_{i}(x)
$$

Consider $r$ statistics

$$
\hat{p}_{i}=\frac{1}{n} \sum_{k=1}^{n} F i\left(x_{k}\right) \quad(i=1, \cdots, r)
$$

and define $\Delta_{i j}$ by

$$
\Delta_{i j}=\int F_{i} d F_{j}-\frac{1}{2}
$$

Now, if the prior distribution that must be estimated and the statistics defined in (1.22) are denoted in vector forms by

$$
\boldsymbol{\omega}=\left(\begin{array}{c}
\omega_{1} \\
\vdots \\
\omega_{r}
\end{array}\right), \quad \hat{\boldsymbol{p}}=\left(\begin{array}{c}
\hat{p}_{1} \\
\vdots \\
\hat{p}_{r}
\end{array}\right),
$$

and if a matrix $A$ is defined by 


$$
A=\left(\begin{array}{cccc}
\frac{1}{2} & \frac{1}{2}+\Delta_{12} & \cdots & \frac{1}{2}+\Delta_{1 r} \\
\frac{1}{2}+\Delta_{21} & \frac{1}{2} & \cdots & \frac{1}{2}+\Delta_{2 r} \\
\vdots & \vdots & \ddots & \vdots \\
\frac{1}{2}+\Delta_{r 1} & \frac{1}{2}+\Delta_{r 2} & \cdots & \frac{1}{2}
\end{array}\right)
$$

we obtain

$$
\varepsilon \hat{\boldsymbol{p}}=A \omega,
$$

where $\varepsilon$ denotes the expectation with respect to $F(x)$. Then, if $|A| \neq 0$,

$$
\widehat{\omega}_{\mathbf{1}}=\boldsymbol{A}^{-1} \hat{\boldsymbol{p}}
$$

is an unbiased estimate of $\omega$, where, denotes the transpose of the matrix.

If we define $B$ and $E$ by

$$
B=\left(\begin{array}{cccc}
0 & \Delta_{12} & \cdots & \Delta_{1 r} \\
\Delta_{21} & 0 & \cdots & \Delta_{2 r} \\
\vdots & \vdots & \ddots & \vdots \\
\Delta_{r 1} & \Delta_{r 2} & \cdots & 0
\end{array}\right)
$$

and

$$
\boldsymbol{E}=\left(\begin{array}{rrrr}
1 & 1 & \cdots & 1 \\
1 & 1 & \cdots & 1 \\
\vdots & \vdots & \ddots & \vdots \\
1 & 1 & \cdots & 1
\end{array}\right)
$$

we have $\boldsymbol{A}=\boldsymbol{B}+1 / 2 \boldsymbol{E}$. Since $\boldsymbol{B}$ is the skew symmetric matrix, $|\boldsymbol{B}|=0$ if $r$ is any odd number. However, we have $|\boldsymbol{A}|=|\boldsymbol{B}|$ for any even number $r$. Thus, in the case that $r$ is any even number, we have

$$
\hat{\omega}_{2}=B^{-1}\left(\hat{\boldsymbol{p}}-\frac{1}{2} \boldsymbol{J}\right)
$$

as an unbiased estimate of $\omega$, where $J$ denotes the transpose of $J^{\prime}=(1, \ldots, 1)$.

The variance-covariance matrix of $\hat{\omega}_{1}$ given in (1.27) can be obtained in the following way:

$$
\begin{aligned}
& \varepsilon\left(\hat{\omega}_{1}-\omega\right)\left(\hat{\omega}_{1}-\omega\right)^{\prime} \\
& =A^{-1}\left[\varepsilon(\hat{p}-A \omega)(\hat{p}-A \omega)^{\prime}\right]\left(A^{-1}\right)^{\prime}
\end{aligned}
$$

and 


$$
\begin{aligned}
& \varepsilon(\hat{\boldsymbol{p}}-\boldsymbol{A} \omega)(\hat{\boldsymbol{p}}-A \omega)^{\prime} \\
& =\frac{1}{n}\left(\sum_{\lambda=1}^{r} \omega_{\lambda} \int_{0}^{1}\left(F_{\mu}-\varepsilon F_{\mu}\right)\left(F_{\nu}-\varepsilon F_{\nu}\right) d F_{\lambda}\right), \\
& \mu, \nu=1, \cdots, r,
\end{aligned}
$$

where $\left(f_{\mu \nu}\right)$ denotes an $r \times r$ symmetric matrix such that $f_{\mu \nu}$ is the $(\mu, \nu)$-element and, denotes the transpose of the matrix. Thus, for the variance-covariance matrix of $\hat{\omega}_{1}$, we have

$$
\varepsilon\left(\hat{\omega}_{1}-\omega\right)\left(\hat{\omega}_{1}-\omega\right)^{\prime}=\frac{1}{n} A^{-1} \Sigma\left(A^{-1}\right)^{\prime}
$$

where $\Sigma$ denotes

$$
\begin{gathered}
\Sigma=\left(\sum_{\lambda=1}^{r} \omega_{\lambda} \int_{0}^{1}\left(F_{\mu}-\varepsilon F_{\mu}\right)\left(F_{\nu}-\varepsilon F_{\nu}\right) d F_{\lambda}\right), \\
\mu, \nu=1, \cdots, r .
\end{gathered}
$$

The variance-covariance matrix of $\hat{\omega}_{1}$ can be also calculated in the same way. Then, the following theorem can be immediately obtained.

If $|\boldsymbol{A}| \neq 0$, the $r$-component random vector $\hat{\boldsymbol{\omega}}_{\mathbf{1}}$ given in (1.27) is asymptotically normally distributed with the expected vector $\omega$ and the variance-cavariance matrix (1/n) $A^{-1} \Sigma\left(A^{-1}\right)^{\prime}$ for large $n$, where $A$ and $\Sigma$ are given in (1.25) and (1.33), and $|\boldsymbol{A}|$ is the determinant of $\boldsymbol{A}$.

In the case that $r$ is any even number, the theorem is also true for changing $\hat{\omega}_{1}$ and $(1 / n) \boldsymbol{A}^{-1} \Sigma\left(\boldsymbol{A}^{-1}\right)^{\prime}$ to $\hat{\omega}_{2}$ and $(1 / n) \boldsymbol{B}^{-1} \Sigma\left(\boldsymbol{B}^{-1}\right)^{\prime}$, where $\hat{\boldsymbol{\omega}}_{2}$ and $\boldsymbol{B}$ are given in (1.30) and (1.28).

However, this theorem may be occationally inconvenient to use in usual problems of statistical inference, because $\omega$ is inculded in $\Sigma$. Then, if the other device to evaluate fluctuation of $\hat{\omega}_{1}$ or $\hat{\omega}_{2}$ independently of $\omega$ is obtainable, it will be practically convenient. We gave the evaluation by the probability inequality for the case of $r=2$ and 3 in [9]. We shall deal with the case of $r=4$ here. In the case of $r=4, \hat{\omega}_{2}$ is

$$
\begin{aligned}
& \hat{\omega}_{1}=\frac{1}{\Delta^{(4)}}\left\{-\left(\hat{p}_{2}-\frac{1}{2}\right) \Delta_{43}-\left(\hat{p}_{3}-\frac{1}{2}\right) \Delta_{24}+\left(\hat{p}_{4}-\frac{1}{2}\right) \Delta_{23}\right\}, \\
& \hat{\omega}_{2}=\frac{1}{\Delta^{(4)}}\left\{\left(\hat{p}_{1}-\frac{1}{2}\right) \Delta_{43}-\left(\hat{p}_{4}-\frac{1}{2}\right) \Delta_{13}-\left(\hat{p}_{3}-\frac{1}{2}\right) \Delta_{41}\right\}, \\
& \hat{\omega}_{3}=\frac{1}{\Delta^{(4)}}\left\{\left(\hat{p}_{4}-\frac{1}{2}\right) \Delta_{12}+\left(\hat{p}_{1}-\frac{1}{2}\right) \Delta_{24}+\left(\hat{p}_{2}-\frac{1}{2}\right) \Delta_{41}\right\},
\end{aligned}
$$

and 


$$
\hat{\omega}_{4}=\frac{1}{\Delta^{(4)}}\left\{-\left(\hat{p}_{3}-\frac{1}{2}\right) \Delta_{12}+\left(\hat{p}_{2}-\frac{1}{2}\right) \Delta_{19}-\left(\hat{p}_{1}-\frac{1}{2}\right) \Delta_{23}\right\},
$$

where $\Delta^{(4)}=\Delta_{12} \Delta_{43}+\Delta_{13} \Delta_{24}+\Delta_{23} \Delta_{41}$. We can assume that $\Delta^{(4)} \geqq 0$, without loss of generality. Then, we have the following.

If $\Delta^{(4)}>0$, we have

$$
P\left(\left|\hat{\omega}_{i}-\omega_{i}\right|<t \Delta^{(4)-1}\right) \geqq 1-2 e^{-(8 / 9)^{n / 2}},
$$

$i=1, \ldots, 4$, for $t>0$.

Attempts to introduce prior knowledges in statistical inference in order to express the corresponding experimental situations seem to be quite natural because experiments are impossible in perfect ignorance. But the difficulty arises because of finding out the adequate measure in order to express various degrees of imperfect knowledge since the necessity of experiments are usually found between a more informative stiuation than ignorance and a less informative one than perfectness.

Therefore, I think that it is usually unable to regard a method as more skillful than others and methods must be examined in connection with applications practically. Then, the empirical Bayes procedure is convenient for preliminary tests if possible.

\section{Acknowledgement:}

The author is indebted to Dr. S. Ikeda for valuable suggestions. The author wishes to thank Miss. Y. Sato for her assistance in preparing manuscript.

\section{References}

[1] Barnard, G.A. (1954), "Sampling inspection and statistical decisions", J.R. Statist. Soc., B, vol. 16, 151-174.

[2] Box, G.E.P. and Tiao, G.C. (1962), "A further look at robustness via Bayes' theorem", Biometrika, vol. 49, 419-432.

[3] Box, G.E.P. and Tiao, G.C. (1964), "A not on criterion robustness and inference robustness, "Biometrika, vol. 51, 169-173.

[4] Fisher, R.A. (1956), Statistical Methods and Scientific Inference, Edinburgh; Oliver and Boyd.

[5] Fraser, D.A.S. (1961), "The fiducial method and invariance", Biometrika, vol. 48, 261-280.

[6] Good, I.J. (1950), Probability and the Weighing of Eridence, London; Griffin.

[7] Good, I.J. (1965), The Estimation of Probabilities, Cambridge (Mass.); M.I.T. .

[8] Hudimoto, H. (1966), "On Bayesian inference", J. Japan Ass. Phil. Sci., vol. 8, 34-41.

[9] Hudimoto, H. (1968), "On the empirical Bayes procedure (1)", Ann. Inst. Statist. Math., vol. 20, 169-185.

[10] Hudimoto, H. (1969), "The estimation of the prior probability distribution and Bayesian inference", J. Japan Ass. Phil. Sci., vol. 9, 129-133.

[11] Hudimoto, H. (1970), "On estimating the prior distribution and discriminating between a mixed sample and a simple sample", (to appear in Symp. Inst. Statist. Math., vol. 2). 
[12] Jeffreys, H. (1961), Theory of Probability, 3rd edition, Oxford; Clarendon Press.

[12] Keynes, J.M. (1921), Treatise on Probability, London; Macmillian.

[14] Lindley, D.V. (1958), "Fiducial distributions and Bayes' theorem", J.R. Statist. Soc., B, vol. 20, 102-107.

[15] Lindley, D.V. (1965), Introduction to Probability and Statistics, Part 2, Cambridge Univ. Press.

[16] Neyman, J. (1962), "Two breakthroughs in the theory of statistical decision making", Rev. Int. Statist. Inst. 30, 11-27.

[17] Plackett, R.L. (1966), "Current trends in statistical inference", J.R. Statist. Soc., A, vol. 129, 249-266.

[18] Pratt, J.W. (1965), "Bayesian interpretation of standard inference", J.R. Statist. Soc., B, vol. 27, 169-184.

[19] Raiffa, H. and Schlaifer, R. (1961), Applied Statistical Decision Theory, Boston; Harvard Graduate School of Business Administration.

[20] Ramsey, F.P. (1931), The Foundations of Mathematics and Other Logical Essays, London; Kegan Paul.

[21] Robbins, H. (1950), "Asymptotically subminimax solutions of compound statistical decision problems", Proc. Second Berkeley Symp. on Math. Statist. and Pro., Univ. of California Press, New York, 131-148.

[22] Hannan, J.F. and Robbins, H. (1955), "Asymptotic solutions of the compound decision problem for two completely specified distributions", Ann. Math. Statist., vol. 26, 3751 .

[23] Robbins, H. (1955), "An empirical Bayes' approach to statistics", Proc. Thrid Berkely Symp. on Math. Statist. and Prob., Univ. of California Press, New York, 157-164.

[24] Robbins, H. (1964), "The empirical Bayes approach to statistical decision problem", Ann. Math. Statist., vol. 35, 1-20.

[25] Savage, L.J. (1954), The Foundations of Statistics, New York; Wiley.

[26] Savage, L.J. et al. (1962), The Foundations of Statistical Inference, London; Methuen.

[27] Welch, B.L. and Peers, H.W. (1963), "On formulae for confidence points based on integrals of weighted likelihoods", J.R. Statist. Soc., B, 25, 318-329.

[28] Welch, B.L. (1965), "On comparisons between confidence point procedures in the care of a single parameter", J. R. Statist. Soc., B, 27, 1-8. 